\title{
Caractérisation Qualitative Et Quantitative De La Consommation De Différents Groupes D'aliments En Côte d'Ivoire
}

\author{
Béhibolo Antoinette Yoboué (Doctorante) \\ Arsène Landry Igor Nogbou (Docteur)
}

Institut National Polytechnique Félix Houphouët-Boigny/ Laboratoire des Procédés Industriels, de Synthèse et de l'Environnement et des Energies nouvelles (LAPISEN), Yamoussoukro, Côte d'Ivoire

Kwadjo Anicet Luc Déré (Maitre-Assistant)

Université Alassane Outtara/ Laboratoire de Biochimie Médical, Unité de Formation et de Recherche Médicale, Bouaké, Côte d'Ivoire

Zita Essan Bla N'Goran-Aw (Maitre-Assistant) Doudjo Soro (Maitre-Assistant)

Institut National Polytechnique Félix Houphouët-Boigny/ Laboratoire des Procédés Industriels, de Synthèse et de l'Environnement et des Energies nouvelles (LAPISEN), Yamoussoukro, Côte d'Ivoire

\section{Gnomblesson Georges Tiahou (Professeur Titulaire)}

Université Alassane Outtara/ Laboratoire de Biochimie Médical, Unité de

Formation et de Recherche Médicale, Bouaké, Côte d'Ivoire

Nogbou Emmanel Assidjo (Professeur Titulaire)

Institut National Polytechnique Félix Houphouët-Boigny, B.P. 1093

Laboratoire des Procédés Industriels, de Synthèse et de l'Environnement et des Energies nouvelles (LAPISEN), Yamoussoukro, Côte d'Ivoire

Doi:10.19044/esj.2018.v14n17p297 URL:http://dx.doi.org/10.19044/esj.2018.v14n17p297

\begin{abstract}
Qualitative and quantitative study of food is essential to highlight causes of nutritional problems in order to evaluate and propose relevant interventions. To achieve this objective, a transversal survey requiring tools to estimate the quantity of food consumed per person was carried out. 417 people were interviewed in seven districts of Côte $d$ 'Ivoire. The results of the survey showed that the average individual dietary diversity score of the respondents was 4.4 . Food groups daily consumed by nearly $50 \%$ of the population are : spices, condiments, beverages (100\%); fish and seafood (93.95\%), oils and fats $(93.83 \%)$, cereals $(89.97 \%)$, roots and tubers $(87.27 \%)$ and other vegetables $(54.07 \%)$. Staple foods were consumed at an
\end{abstract}


average of $450.00 \pm 225.00$ and $562.50 \pm 290.50 \mathrm{~g}$ per meal per person. Rice $(33.66 \pm 6.06 \%)$ and cassava $(23.09 \pm 5.28 \%)$ were the most consumed staple foods. In sum, the choice of the type and quantity of staple foods to be consumed per individual should be guided by the activity perfomed, at the risk of increasing the morbidity and mortality rate related to metabolic diseases.

Keywords: Quantitative, qualitative, groups, foods

\section{Résumé}

L'étude qualitative et quantitative de l'alimentation est indispensable pour mettre en lumière les causes des problèmes nutritionnels en vue d'évaluer et proposer des interventions pertinentes. Pour atteindre cet objectif une enquête transversale nécessitant des outils d'estimation de la quantité des aliments consommés par personne a été réalisée. Elle s'est déroulée auprès de 417 personnes dans sept chefs-lieux de district de la Côte d' Ivoire. Les résultats de l'enquête ont montré que le score de diversité alimentaire individuel moyen des personnes interrogées était de 4,4. Les groupes d'aliments journalièrement consommé par près de $50 \%$ de la population sont: les épices, condiments, boissons (100\%); poissons et fruits de mer $(93,95 \%)$, huiles et graisses $(93,83 \%)$, céréales $(89,97 \%)$, racines et tubercules $(87,27 \%)$ et autres légumes $(54,07 \%)$. Les aliments de base étaient consommés à des quantités moyennes de $450,00 \pm 225,00$ et 562,50 \pm $290,50 \mathrm{~g}$ par repas et par personne. Le riz $(33,66 \pm 6,06 \%)$ et le manioc $(23,09 \pm 5,28 \%)$ étaient les aliments de base les plus consommés. En somme, le choix du type et de la quantité d'aliments de base à consommer par individu, doit être orienté par l'activité effectué au risque d'augmenter le taux de morbidité et de mortalité lié aux maladies métaboliques.

Mots clés : quantitative, qualitative, groupes, aliments

\section{Introduction:}

L'alimentation est un besoin fondamental chez l'homme, puisqu'elle apporte à son organisme les nutriments et l'énergie dont il a besoin pour se construire et fonctionner (Rigaux, 2014). Dans les pays en développement comme dans ceux développées, la surveillance de la qualité de l'alimentation est primordiale, tant les formes de malnutrition sont nombreuses et leurs conséquences mesurables en terme de vies humaines (Becquey, 2006).

De ce fait, les politiques de santé publique visant la réduction des maladies nutritionnelles examinent de plus en plus le comportement alimentaire des populations à travers des enquêtes. En effet, les enquêtes alimentaires sont des méthodes développées pour évaluer les apports 
alimentaires d'un individu ou d'un groupe d'individus. En épidémiologie, elles permettent d'étudier les relations entre l'alimentation et certaines pathologies afin d'identifier des nutriments, des aliments ou des profils de consommation bénéfiques ou néfastes pour la santé. En clinique, l'évaluation des apports alimentaires fait partie de la prise en charge thérapeutique des maladies dites «liées à la nutrition» (Anonyme 1, 2011). Malgré les différentes actions d'interventions des organismes nationaux et internationaux (CNN, PAM, PNN, $\mathrm{ACF})$, les difficultés liées à l'alimentation restent préoccupantes.

En Côte d'Ivoire, plusieurs études concernant l'alimentation et la nutrition ont été menées (Kouassi et al. 2013; Moyabi et Affeli, 2013; et Anonyme 2, 2016). Ces travaux ont porté sur la vulnérabilité alimentaire, la situation nutritionnelle et le niveau de sécurité alimentaire en Côte d' Ivoire. Ces différentes études ont permis d'identifier les principaux problèmes de nutrition dans le pays et les obstacles à l'accroissement des actions en faveur de la nutrition.

Cependant, des données sur le mode d'alimentation des adultes au niveau national, la détermination des quantités d'aliments de base consommées et leurs apports énergétiques journalier sont très peu disponibles en Côte d'Ivoire. Par ailleurs, des outils tels que les photographies pour le suivi et l'évaluation quantitative de la consommation des aliments sont quasi inexistants.

L'objectif de ce travail est de déterminer le profil alimentaire et l'état nutritionnel des populations en Côte d'Ivoire. Pour atteindre cet objectif, des enquêtes de rappel qualitatif et quantitatif des dernières 24 heures ont été menées dans plusieurs chefs-lieux de districts de la Côte d'Ivoire. De telles données sont indispensables pour l'information de la population, la mise en place de politiques alimentaires adaptées, l'évaluation les politiques existantes, de même que pour leur utilisation dans le cadre de la recherche scientifique.

\section{Matériel et méthodes}

\section{Zone d'étude}

Les enquêtes se sont déroulées de Novembre 2016 à Février 2017 dans sept villes de la Côte d'Ivoire qui sont: Abidjan, Abengourou, Dabou, Daloa, Korhogo, Man et Yamoussoukro. Ces villes sont des chefs-lieux de district. Elles ont été choisies dans le but de décrire la diversité alimentaire individuelle et la fréquence de consommation des différents groupes d'aliments dans les régions du Nord, Sud, Centre, Est et Ouest de la Côte d'Ivoire. 


\section{Type d'étude}

Dans le cadre de ce travail il s'est agit d'une enquête alimentaire décrite par le rappel qualitatif et quantitatif des dernières 24 heures. C'est une enquête rétrospective et transversale dans laquelle la population cible avait au moins 18 ans.

\section{Population}

Le choix des personnes qui ont participé à l'enquête a été fait par la technique de l'échantillonnage par sondage systématique. Le nombre de personnes interrogées était de 417 déterminés selon l'expression de Giezendanner (2012). Le choix du nombre d'individus dans chaque chef-lieu de district a été proportionnel à la taille démographique de chaque ville (échantillonnage par quota) et le nombre d'individus par chef-lieu de district est présenté au tableau 1.

$$
\boldsymbol{n}=\boldsymbol{t}^{2} \boldsymbol{x} \frac{p(1-p)}{e^{2}} \quad \text { (Giezendanner, 2012) }
$$

Avec $\mathbf{n}$ : la taille de l'échantillon, e: la marge d'erreur, t: le coefficient de marge déduit du taux de confiance, p: la proportion des éléments de la population-mère qui présente une propriété donnée. Tableau 1: Nombres de personnes enquêtées par zone d'étude

\begin{tabular}{|cr|}
\hline Chefs-lieux de districts & $\begin{array}{c}\text { Nombres de personnes } \\
\text { enquêtées }\end{array}$ \\
\hline Abidjan & 257 \\
Abengourou & 20 \\
Dabou & 16 \\
Daloa & 43 \\
Korhogo & 35 \\
Man & 24 \\
\hline Yamoussoukro & 22 \\
\hline
\end{tabular}

\section{Rappel qualitatif}

L'enquête consistait à recenser, en un jour excepté le jour de festivité, tous les plats, sauces, snacks (grignotages) et boissons consommés la veille. Cette évaluation de diversité alimentaire se déroule en deux étapes.

La première étape consiste à effectuer un rappel de consommation alimentaire de 24 heures. Cette méthode consiste à enregistrer l'ensemble des aliments et des boissons consommés par la personne interrogée durant les 24 heures ayant précédé l'interview (Biro et al., 2002). Selon la technique de Johnson et al. (1996); l'interrogatoire est guidé par une série de questions qui portent spécifiquement sur certains points source d'erreurs et une liste de groupes d'aliments. Dans le cadre de ce travail, la liste de groupes d'aliments 
(16 groupes) retenu pour la réalisation de 1'enquête était celle basée sur la subdivision des groupes d'aliments effectuée par la FAO (Kennedy et al., 2013) .

Au cours de cette interrogation, les aliments et boissons consommés sont identifiés, soulignés dans la liste où figure chaque groupe d'aliments. Puis, l'on affecte le coefficient 1 dans la colonne en regard de chaque groupe dont au moins un des éléments a été souligné ou inscrire « 0 » dans cette colonne lorsqu'il est avéré qu'aucun aliment de ce groupe n'a été consommé.

La deuxième étape consiste à calculer le Score de Diversité Alimentaire Individuel (SDAI) nécessitant la fusion des 16 groupes d'aliments utilisés pour les enquêtes en 9 groupes (Kennedy et al., 2013). Il s'agit de faire la somme de tous les coefficients des colonnes en regard de chaque groupe dont au moins un des éléments a été souligné. Cette addition est effectuée pour chaque individu enquêté et correspond au SDAI.

\section{Rappel quantitatif}

Le rappel quantitatif des dernières 24 heures a permis de déterminer les quantités d'aliment consommées par personne et par repas. Cette méthode est employée pour évaluer les apports énergétiques et nutritionnels (Lawn et Harvey, 2003). Il s'agit d'effectuer un entretien pendant lequel on demande au sujet de se remémorer et de décrire les quantités d'aliments et boissons consommées pendant les 24 heures précédentes. Dans le cadre de ce travail, les informations sur l'appréciation quantitative ont été obtenues à l'aide de photographies des aliments de bases locaux repérés au cours d'une pré-enquête (photographies en annexe). Ces aliments de base (riz, mais, igname, manioc, patate douce, banane plantain) ont été cuits à l'eau puis pesés à différentes masse $(225 \mathrm{~g}, 450 \mathrm{~g}, 675 \mathrm{~g}$, $900 \mathrm{~g})$. Ainsi, les informations obtenues par pesée ont été mis sur support photographique dans le but de les présenter aux répondants afin qu'ils choisissent les quantités d'aliments de base consommées par repas.

- Détermination de la valeur énergétique des aliments de base

La valeur énergétique (kilocalories / $100 \mathrm{~g}$ d'aliment) a été déterminée en utilisant les coefficients d'Atwater et Benedict (1902).

Valeur énergétique $(\mathrm{Kcal})=[($ Glucides $(\%) \times 4)+($ Protéines $(\%) \times 4)+($ Lipides $(\%) \times 9)]$

\section{Analyse statistique}

Les données obtenues à partir de ces enquêtes ont été analysées à l'aide du logiciel Excel et STATISTICA version 7.1. 


\section{Résultats et Discussion Evaluation qualitative}

L'évaluation qualitative de la diversité alimentaire concerne le Score de Diversité Alimentaire Individuel (SDAI) et la fréquence journalière de consommation des 16 groupes d'aliments.

\section{Evaluation Score de Diversité Alimentaire Individuel (SDAI)}

La figure 1 décrit le SDAI moyen de la population des chefs-lieux de districts qui est de 4,38 $\pm 1,21$. Ce SDAI moyen est différent de ceux déterminés par Déré et al. (2016) qui étaient de 5,6 $\pm 1,58$ et de $6 \pm 1$ respectivement chez des personnes diabétiques et hypertendues. Cette différence pourrait être due à l'état de santé des personnes enquêtées. En effet, les participants à cette présente investigation étaient en bonne santé apparente. Par contre, ceux interrogés dans l'étude de Déré et al. (2016) sont atteints de maladies chroniques. Ceci révèle que la diversité alimentaire individuelle de ces patients a pu être influencée par les conseils médicaux reçus.

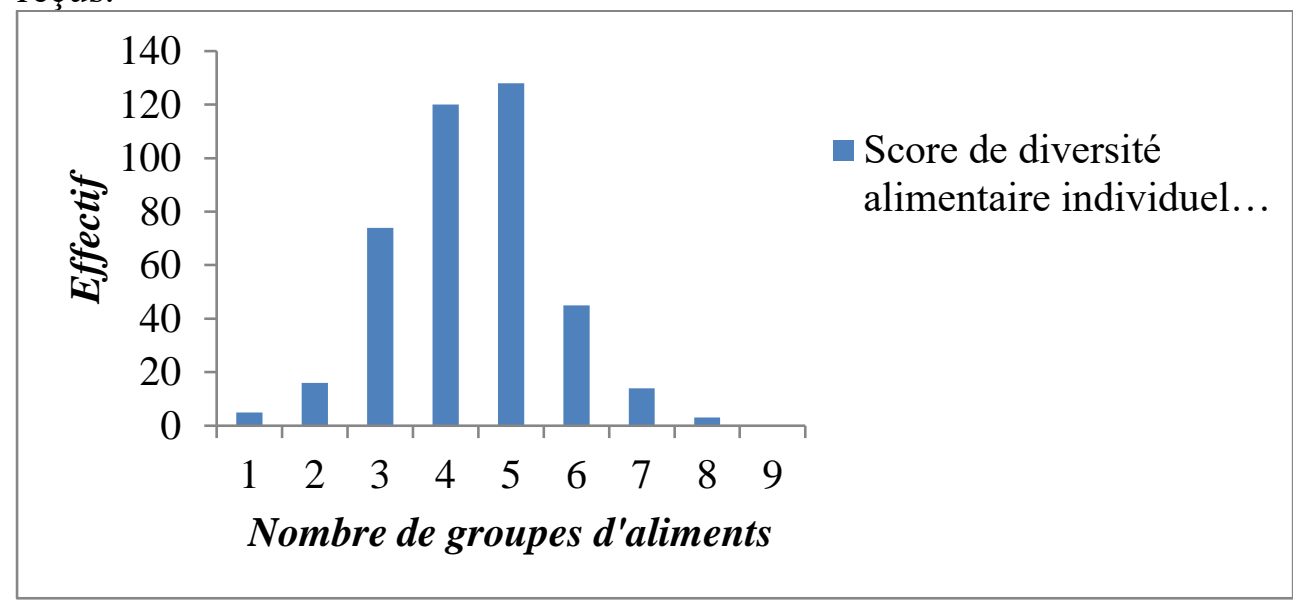
(SDAI)

Figure 1: Distribution du Score de Diversité Alimentaire Individuel

La subdivision de ce score en tercile (Tableau 2), montre que 23,46 $\%$ de la population a consommée moins de quatre groupes d'aliments différents en 24 heures. Ces différents groupes d'aliments définissent le profil alimentaire des personnes investiguées. Le profil alimentaire de ce groupe est composé de céréales, des huiles et graisses, des épices, condiments, boissons et présente une diversité alimentaire faible. La diversité alimentaire moyenne est observée chez 29,63\% de la population. Cela signifie qu'ils ont consommés en moyenne quatre groupes d'aliments différents pendant 24 heures c'est-à-dire qu'en plus des céréales, huiles et graisses, épices, condiments et boissons leur profil alimentaire est aussi constitué de poissons et fruits de mer. Par contre, elle est élevée chez 46,91 
$\%$ de la population enquêtée. Ces individus ont consommés plus de quatre groupes d'aliments différents au cours des 24 heures précédant l'enquête. Le profil alimentaire de ce groupe est composé de céréales, racines et tubercules, autres légumes, poissons et fruits de mer, huiles et graisses, sucreries et des épices, condiments, boissons. Au total, 53,09 \% de la population enquêtée expriment une diversité alimentaire non satisfaisante. Ce résultat est identique à celui rapporté par Kouassi et al. (2013). Ces auteurs indiquaient que $52,10 \%$ de la population dans les zones urbaines d'Abidjan avaient une diversité alimentaire non satisfaisante. Le niveau de diversité alimentaire des populations n'a pas substantiellement évolué entre 2013 et 2017.

Tableau 2: Profils alimentaires des populations étudiées par tertile de diversité alimentaire

\begin{tabular}{|c|c|c|}
\hline NIVEAUX DE SCORE & $\begin{array}{c}\text { PROPORTION DE LA } \\
\text { POPULATION }\end{array}$ & PROFILS ALIMENTAIRES \\
\hline $\begin{array}{c}\text { Score de Diversité } \\
\text { alimentaire faible (<4) }\end{array}$ & $23,46 \%$ & $\begin{array}{c}\text { Céréales, huiles et graisses, } \\
\text { épices, condiments, boissons. }\end{array}$ \\
\hline $\begin{array}{c}\text { Score de Diversité } \\
\text { alimentaire moyenne (=4) }\end{array}$ & $29,63 \%$ & $\begin{array}{c}\text { Céréales, poissons et fruits de } \\
\text { mer, huiles et graisses, épices, } \\
\text { condiments, boissons. }\end{array}$ \\
\hline $\begin{array}{c}\text { Score de Diversité } \\
\text { alimentaire élevé }(>\mathbf{4})\end{array}$ & $\begin{array}{c}\text { Céréales, Racines et tubercules, } \\
\text { autres légumes, poissons et } \\
\text { fuits de mer, huiles et graisses, } \\
\text { sucreries, épices, condiments, } \\
\text { boissons. }\end{array}$ \\
\hline
\end{tabular}

Les SDAI de la population des sept (7) chefs-lieux de district présenté par le Tableau 3 révèle que cette population a une moyenne de diversité alimentaire comprise entre 3,43 et 5,87. Le SDAI le moins élevé est observé à Korhogo $(3,43)$ tandis que la ville d'Abidjan $(5,87)$ présente le SDAI le plus élevé. De plus, l'analyse statistique des SDAI décrit une différence significative entre les SDAI des villes. Cela souligne que la diversité alimentaire diffère d'une zone à l'autre. De même, le test post hoc de Fisher permet de regrouper les Sept (7) chefs-lieux de district en trois (3) groupes. Le premier groupe, renferme les populations des villes de Korhogo $(3,43 \pm 1,24)$, Man $(3,80 \pm 1,15)$ et Abengourou $(3,95 \pm 1,26)$ qui ont les diversités alimentaires individuelles les plus faibles. Yamoussoukro $(4,40 \pm$ $1,32)$, Daloa $(4,45 \pm 1,15)$ et Dabou $(4,77 \pm 0,94)$ présentent des SDAI intermédiaire. La diversité alimentaire la plus élevée est observée auprès des populations d'Abidjan $(5,87 \pm 1,13)$. Les villes dont les populations présentent les SDAI plus faibles sont situées respectivement au Nord, à l'Est et à l'Ouest de la Côte d'ivoire. Les individus dans ces zones consomment environ trois (3) à quatre (4) groupes d'aliments différents en 24 heures. Ils présentent un régime alimentaire moins diversifié que les populations des autres chefs-lieux de districts. Cette faible diversité alimentaire dans ces 
zones serait due aux problèmes de disponibilité, d'accessibilité et à l'attachement aux coutumes alimentaires qui sont des éléments pouvant fragiliser la diversité alimentaire. Ce niveau de diversité alimentaire moins élevé pourrait être une des causes des taux les plus élevés de retard de croissance dans ces zones Nord (39\%), Nord-Est (39\%), Nord-Ouest (32\%) et Ouest (34\%) de la Côte d'Ivoire. Ainsi, dans ces zones, les taux de retard de croissance sont supérieurs à $30 \%$, avec des profils de malnutrition chronique avérés (CNN, 2014). Par ailleurs, la population de la ville d'Abidjan présente une alimentation plus diversifiée. Selon la classification de l'OMS, les populations de la ville d'Abidjan, ont une valeur de retard de croissance inférieure à $20 \%$, donc jugé acceptable (CNN, 2014).

Tableau 3: SDAI par chefs-lieux de districts

\begin{tabular}{|cc|}
\hline Chefs-lieux de districts & Score de Diversité Alimentaire Individuel (SDAI) \\
\hline Korhogo & $3,43 \pm 1,24^{\mathbf{a}}$ \\
\hline Man & $3,80 \pm 1,15^{\mathbf{a}}$ \\
\hline Abengourou & $3,95 \pm 1,26^{\mathbf{a}}$ \\
\hline Yamoussoukro & $4,40 \pm 1,32^{\mathbf{b}}$ \\
\hline Daloa & $4,45 \pm 1,15^{\mathbf{b}}$ \\
\hline Dabou & $4,77 \pm 0,94^{\mathbf{b}}$ \\
\hline Abidjan & $5,87 \pm 1,13^{\mathbf{c}}$ \\
\hline
\end{tabular}

Les valeurs en colonne affectées de la même lettre ne sont pas significativement différentes avec le test de Fisher au seuil de $5 \%$.

\section{Fréquence de consommation des $\mathbf{1 6}$ groupes d'aliments}

La figure 2 présente la distribution de la fréquence de consommation journalière des différents groupes d'aliments. Elle révèle d'abord que le groupe d'aliment composé d'épices (poivre, sel), condiments (sauce de soja, sauce piquante) et boissons (café, thé, boissons alcoolisées) a été utilisé par l'ensemble des personnes enquêtées (100\%). Ensuite, les poissons et fruits de mer ainsi que les huiles et graisses ont été consommés à des fréquences respectives de $93,95 \%$ et $93,83 \%$. Les fréquences de consommation des céréales, racines et tubercules ont été respectivement de 89,97\% et 87,27\%. Les groupes d'aliments tels que : "autres légumes", "sucreries", "autres fruits" et "légumineuse, noix, graines" sont consommés à des fréquences respectivement $54,07 \% ; 31,52 \% ; 29,58 \%$ et $29,34 \%$. Avec une fréquence de $22,32 \%$, le groupe "fruits riches en vitamine A" a été plus consommé que le groupe "viande", consommé à une fréquence journalière de 18,55\%. Le groupe "lait et produits laitiers" vient après le groupe "viande", avec une fréquence de consommation de 16,03\%. Il est suivi des groupes d'aliments "légumes feuilles verts foncés", "œufs", et "abats" qui ont respectivement des fréquences de consommation de $14,75 \%, 10,63 \%$ et $3,20 \%$. Enfin, le 
groupe légumes et tubercules riches en vitamine A a été moins consommé avec une fréquence de $0,33 \%$.

Les céréales, racines et tubercules, sont les principales cultures alimentaires destinées à la consommation humaine (Sanginga et Mbabu, 2015). Parmi ces aliments de base, les céréales constituent les aliments énergétiques les plus consommés dans les zones étudiées. Cette consommation des céréales a été également confirmée par les travaux de Macauley et Ramadjita (2015) qui mettaient en évidence que les céréales telles que le sorgho, le millet, le blé, le maïs et le riz sont les aliments de base essentiels pour la majorité de la population du Sénégal. Les potagères telles que l'aubergine, gombo, la tomate, l'oignon et ceux disponibles localement sont les légumes généralement utilisés comme ingrédient dans la réalisation des sauces pour l'accompagnement des aliments de base. Généralement, ces individus confectionnent les plats avec de l'huile, la graisse (origine animale et végétale) ainsi que des épices et condiments (poivre, sel, sauce de soja, sauce piquante). Par ailleurs, les poissons et fruits de mer représentent la première source de protéine animale de ces populations urbaines. En fait, les poissons sont faciles d'accès et disponibles à toutes les bourses. Cette consommation de poissons est également mise en évidence par les résultats d'enquête réalisée par l'Ocean World Academy of Japan (OWAJ) en 2016. Selon Shiraishi et al. (2016), la consommation des produits halieutiques en Cote d'Ivoire est en constante augmentation chaque année. L'analyse de la fréquence de consommation des aliments a permis d'identifier les aliments faiblement consommés et les plus utilisés par la population. Les 16 groupes d'aliments sont consommés à des fréquences relativement variées. Les légumes et tubercules riches en vitamine $\mathrm{A}$ sont très peu fréquents dans la composition des plats, par contre, les épices, condiments et boissons font partie de l'alimentation quotidienne de ces populations enquêtées. 
Epices, condiments, boissons

Poisson et fruits de mer

Huiles et graisses

Céréales

Racines et tubercules

Autres légumes

Sucreries

Autres fruits

Légumineuse, noix, graines

Fruits riches en vitamine A

Viande

Lait et produits laitiers

Légumes feuilles verts foncés

Eufs

Abats
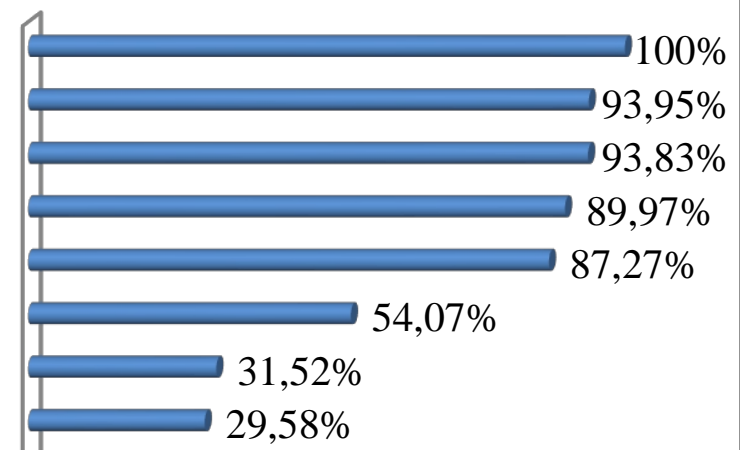

I

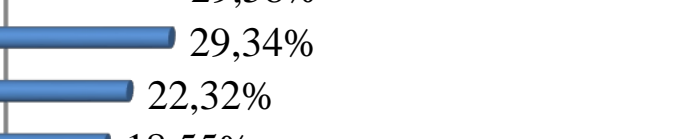

Légumes et tubercules riches en... $0,33 \%$

Figure 2 : Distribution de la fréquence de consommation des 16 groupes d'aliments

Toutefois, la fréquence de consommation de ces aliments ne met pas en évidence les quantités d'aliments de base (représentent la première source d'énergie des consommateurs) consommées. Ceux-ci ont été évalués à partir de la détermination des quantités d'aliments de base consommées (rappel quantitatif) et du calcul d'apport énergétique journalier.

\section{Evaluation quantitative de la diversité alimentaire}

Le Tableau 4 révèle les quantités moyennes d'aliments de base consommées par la population dans chaque chef-lieu de district. Les populations des sept zones étudiées ont des consommations moyennes identiques en riz et en manioc. Le maïs est plus consommé à Korhogo (562,5 $\pm 129,90 \mathrm{~g}$ par repas et par jour) mais cette consommation est quasi nulle à Abengourou $(0,00 \pm 0,00 \mathrm{~g}$ par repas et par jour). L'igname est en moyenne plus consommée par repas et par jour par les populations des zones d'Abidjan (357,64 $\pm 225,86$ g), Abengourou $(375,00 \pm 129,90 \mathrm{~g})$, Dabou $(337,50 \pm 129,90 \mathrm{~g})$, Daloa $(375,00 \pm 116,19 \mathrm{~g})$ et Yamoussoukro $(375,00 \pm$ $129,90 \mathrm{~g})$. Par contre, ce tubercule est moins consommé par les populations des régions de Man $(225,00 \pm 0,00 \mathrm{~g})$ et Korhogo $(0,00 \pm 0,00 \mathrm{~g})$. La banane plantain généralement sous la forme «foutou» est plus présente journalièrement dans les mets des habitants des régions d'Abidjan $(419,06 \pm$ 
$163,10 \mathrm{~g})$ et d'Abengourou $(417,86 \pm 185,04 \mathrm{~g})$ mais très peu consommé par la population de Korhogo $(0,00 \pm 0,00 \mathrm{~g})$.

Selon Ruf (2010), la consommation des aliments de base en Côte d'Ivoire était repartie par région; le centre avait un système alimentaire privilégiant l'igname, le Nord et l'Ouest favorisaient plus la consommation du maïs et du riz. Le test post hoc de Fisher des quantités d'aliments de base consommées nous indiquent que les populations du Nord, conservent toujours leur alimentation plus orientée vers les céréales surtout le maïs. Egalement, le peuple de l'Est (district d'Abengourou), qui consommait autrefois plus les racines (igname) (Dubresson, 1989) et la banane plantain, reste toujours attaché à leur coutume alimentaire qui est la consommation plus accentuée de l'igname et des fruitiers tel que la banane plantain. De même, la population du Centre (district de Yamoussoukro) conserve toujours l'igname comme première source énergétique dans leur alimentation. Cependant, la consommation du riz et du manioc semble être la même pour l'ensemble des populations étudiées. En effet, le riz qui est un aliment énergétique disponible à toute saison, est de plus en plus utilisé comme substitut des aliments de base des différentes régions de la Côte d'Ivoire qui sont moins conservables. Par ailleurs, les tubercules de manioc sont transformés en différents produits alimentaires tels que l'attiéké, le gari, le tapioca et la liqueur. Cette amélioration permet une conservation plus ou moins longue de ces produits (N'Zué et al., 2013).

Tableau 4 : Quantité moyenne (g) d'aliment de base consommé par chef-lieu de district

\begin{tabular}{ccccrr}
\hline & Riz & Maïs & Igname & Manioc & $\begin{array}{c}\text { Banane } \\
\text { plantain }\end{array}$ \\
\hline Abidjan & $428,99 \pm$ & $478,13 \pm$ & $357,64 \pm$ & $323,32 \pm$ & $419,06 \pm$ \\
& $142,80^{\mathbf{a}}$ & $187,77^{\mathbf{b}}$ & $225,86^{\mathbf{b}}$ & $148,31^{\mathbf{a}}$ & $163,10^{\mathbf{c}}$ \\
Abengourou & $436,76^{\mathbf{b}} \pm$ & $0,00 \pm$ & $375,00 \pm$ & $337,50 \pm$ & $417,86 \pm$ \\
& $94,88^{\mathbf{a}}$ & $0,00^{\mathbf{a}}$ & $129,90^{\mathbf{b}}$ & $225,00^{\mathbf{a}}$ & $185,04^{\mathbf{c}}$ \\
Dabou & $412,50 \pm$ & $450,00 \pm$ & $337,50 \pm$ & $365,63 \pm$ & $380,77 \pm$ \\
& $129,90^{\mathbf{a}}$ & $125,00^{\mathbf{b}}$ & $129,90^{\mathbf{b}}$ & $206,13^{\mathbf{a}}$ & $108,08^{\mathbf{b c}}$ \\
Daloa & $450 \pm$ & $337,50 \pm$ & $375,00 \pm$ & $265,50 \pm$ & $375,00 \pm$ \\
& $85,04^{\mathbf{a}}$ & $129,90^{\mathbf{b}}$ & $116,19^{\mathbf{b}}$ & $96,78^{\mathbf{a}}$ & $159,10^{\mathbf{b c}}$ \\
Korhogo & $495 \pm$ & $562,5 \pm$ & $0,00 \pm$ & $315,00 \pm$ & $0,00 \pm$ \\
& $148,20^{\mathbf{a}}$ & $129,90^{\mathbf{c}}$ & $0,00^{\mathbf{a}}$ & $201,25^{\mathbf{a}}$ & $0,00^{\mathbf{a}}$ \\
\hline Man & $423,53 \pm$ & $375,00 \pm$ & $225,00 \pm$ & $302,88 \pm$ & $318,75 \pm$ \\
& $109,14^{\mathbf{a}}$ & $129,90^{\mathbf{b}}$ & $0,00^{\mathbf{a}}$ & $201,25^{\mathbf{a}}$ & $115,86^{\mathbf{b}}$ \\
Yamoussoukro & $450,00 \pm$ & $450,00 \pm$ & $375,00 \pm$ & $286,36 \pm$ & $382,50 \pm$ \\
& $147,30^{\mathbf{a}}$ & $159,10^{\mathbf{b}}$ & $129,90^{\mathbf{b}}$ & $145,50^{\mathbf{a}}$ & $108,69^{\mathbf{b c}}$ \\
\hline
\end{tabular}

Les valeurs en colonne affectées de la même lettre ne sont pas significativement différentes avec le test de Fisher au seuil de $5 \%$.

Les apports énergétiques des quantités moyennes des aliments de base consommées par l'ensemble des populations enquêtées sont répertoriés dans le tableau 5. La quantité moyenne de riz, d'igname et de banane 
plantain cuite consommée par jour et par repas a été de 562,50 $\pm 290,50 \mathrm{~g}$. Ces aliments apportent respectivement 1985,64 $\pm 1025,37 \mathrm{kcal}, 1081,14 \pm$ $558,29 \mathrm{kcal}$ et $658,13 \pm 339,85 \mathrm{kcal}$ par repas par jour; soit $32,91 \%$ à 99,28 $\%$ de couverture des besoins énergétiques pour un adulte dépensant au moins 2000 kcal par jour. Quant à la quantité moyenne de maïs et au manioc ingérée par jour et par repas, elle, était de 450,00 $\pm 225,00 \mathrm{~g}$. Ces aliments de base (manioc, maïs) apportent respectivement 2045,85 $\pm 1056,41$ kcal et $882,35 \pm 439,01$ kcal par jour et par repas. Selon Guy-Grand (2017) le besoin énergétique est de $2100 \mathrm{Kcal} / \mathrm{j}$ pour les femmes et $2600 \mathrm{Kcal} / \mathrm{j}$ pour les hommes. Mais en région tempérée et avec une activité physique moyenne ; ces valeurs peuvent se révéler inférieures ou supérieures aux besoins de certains individus. Ainsi, les apports énergétiques des aliments de base étudiés conviendraient pour les populations ayant des activités physiques intenses surtout pour les consommations moyennes de riz, igname et du maïs. Par contre, ceux exerçants des activités physiques modérées ou qui vivent dans la sédentarité, les apports énergétiques des quantités moyennes de la banane plantain et du manioc sont jugés acceptables pour leur équilibre nutritionnel. Toutefois, les apports énergétiques des quantités moyennes de riz, igname et maïs représentent un danger pour leur santé. Ces apports excessifs constituent l'une des causes de l'accumulation des composés glucidiques (nutriments énergétiques) pouvant entrainer certaines maladies chroniques telles que l'obésité, le diabète de type 2 et l'hypertension artérielle dont les cas ne cessent de croitre en Côte d'Ivoire (N'Dri-Yoman, 2014).

Le riz est l'aliment le plus consommé par l'ensemble des populations rencontré avec une fréquence journalière de 33,66 $\pm 6,05 \%$. Ensuite vient le manioc qui est généralement plus consommé sous deux formes; (attiéké ou placali) $(23,09 \pm 5,28 \%)$. Puis, la banane plantain (bouillie et foutou) qui est consommée journalièrement par $17,70 \%$ de la population enquêtée. Les proportions des populations qui consomment le maïs et l'igname sont respectivement de 5,86 $\pm 6,14 \%, 5,14 \pm 3,86 \%$. Ces aliments de base sont les moins consommés par les populations étudiées. Ces résultats sont différents de ceux rapportés par Sangaré et al. (2009). Selon ces auteurs, l'igname est considérée comme l'aliment ou la culture alimentaire de base la plus importante en Côte d'Ivoire. Cette différence serait due aux zones d'étude. En effet, des travaux réalisés par Bricas et Attaie (2009) ont révélé que l'igname est consommée en moindre quantité en ville qu'en zone rurale. Le Maïs est la céréale la plus cultivée en Côte d'Ivoire après le riz mais la moins consommée par la population investiguée. Cette faible fréquence journalière consommation du maïs par l'ensemble de la population pourrait s'expliquer par le fait que le met généralement confectionné à base de maïs 
est la pâte de mais non fermenté localement appelé «toh » qui serait un met fréquent en milieu urbain.

Les céréales, racines et tubercules sont les aliments de base consommés par 82,30 \% des populations enquêtées dans les zones d'études. Ils représentent les principales sources énergétiques. Nos résultats sont similaires à ceux de Kouamé et Enoh (2011) qui notaient que les sousgroupes «racines et tubercules » et «céréales » contribuent de façon cumulée pour plus de $60 \%$ à la formation des disponibilités calorifiques en Côte d'Ivoire. L'igname et le manioc au niveau des racines et tubercules et le riz pour les céréales, sont les produits qui contribuent le plus à la formation des disponibilités calorifiques par personne par jour au niveau national (Kouamé et Enoh, 2011).

Tableau 5 : Apport énergétique des aliments de base consommé

\begin{tabular}{cccc}
\hline Aliments & $\begin{array}{c}\text { quantité moyenne } \\
\text { consommées/repas/jour } \\
(\mathbf{g})\end{array}$ & $\begin{array}{c}\text { Fréquence de } \\
\text { consommation } \\
\text { journalière } \mathbf{( \% )}\end{array}$ & $\begin{array}{c}\text { Apports } \\
\text { énergétiques } \\
\text { moyens/repas/jour }\end{array}$ \\
\hline Riz & $562,50 \pm 290,50$ & $33,66 \pm 6,05$ & $1985,64 \pm 1025,37$ \\
Maïs & $450,00 \pm 225,00$ & $5,86 \pm 6,14$ & $2045,85 \pm 1056,41$ \\
Igname & $562,50 \pm 290,50$ & $5,14 \pm 3,86$ & $1081,14 \pm 558,29$ \\
Manioc & $450,00 \pm 225,00$ & $23,09 \pm 5,28$ & $882,35 \pm 439,01$ \\
\hline Banane plantain & $562,50 \pm 290,50$ & $17,70 \pm 12,80$ & $658,13 \pm 339,85$ \\
\hline
\end{tabular}

\section{Conclusion:}

Cette étude avait pour objectif d'analyser la consommation qualitative et quantitative des différents groupes d'aliments dans quelques chefs-lieux de district de la Côte d'Ivoire. L'analyse des résultats montre que le score de diversité alimentaire individuel est en moyenne de 4,4. Le sousgroupe renfermant les épices, condiments et boissons est consommé par toute la population. La source de protéine la plus utilisée est le poisson et l'aliment de base le plus consommé est le riz. Les aliments de base couvrent en moyenne près de 658,13 à 2045,85 kcal par repas et par jour. Par ailleurs, d'autres travaux concernant le calcul de la dépense énergétique des individus doivent être effectués dans les zones urbaines et rurales afin de mener à mieux la surveillance de la qualité de l'alimentation en Côte d'Ivoire.

\section{References:}

1. Rigaux, C. (2014). Méthodes de Monte Carlo du second ordre et d'inférence bayésienne pour l'évaluation des risques microbiologiques et des bénéfices nutritionnels dans la transformation des légumes. Institut des Sciences et Industries du Vivant et de l'Environnement. Thèse AgroParisTech. 209 p.

2. Becquey, E. (2006).Validation d'un indicateur de diversité alimentaire par l'adequation du régime à Ouagadougou, Burkina 
Faso. Institut de Recherche pour le Developpement. Mémoire présenté pour l'obtention du Master 2 Recherche. Université Pierre et Marie Curie. 67p.

3. Anonyme1 (Université Médicale Virtuelle Francophone). (2011). Méthodologie des enquêtes alimentaires. Collège des enseignants de nutrition. Support de cours. 28 p.

4. Kouassi, B., N'Goran, P., Tapé, C., Anon, B., Foto, M., Assi, Y., Daouda, S. \& Gbané M. (2013). Enquête sur la vulnérabilité alimentaire en milieu urbain : cas de la ville d'Abidjan. Rapport final du Ministère de l'agriculture et du Ministère de la santé et de la lutte contre le SIDA (Côte d'Ivoire). 57p.

5. Moyabi, S. \& Affeli W. (2013). Evaluation de la sécurité alimentaire à l'Ouest et au Nord. Rapport du Bureau du Ministère de l'Agriculture, Direction des productions vivrières et de la sécurité alimentaire (Côte d'Ivoire). 26p.

6. Anonyme 2 (2016). Plan National Multisectoriel de Nutrition 20162020. Rapport du Ministère de la Santé et de 1'Hygiène Publique. $37 \mathrm{p}$.

7. Giezendanner, F. (2012). Taille d'un échantillon aléatoire et Marge d'erreur. $22 \mathrm{p}$.

8. Biro G., Hulshof K., Ovesen L. \& Amorim C. (2002). Selection of methodology to assess food intake. Eurpean Journal Clinic Nutrition .2,S25-S32.

9. Johnson, R., Driscoll, P. \& Goran M. (1996). Comparison of multiple-pass 24-hour recall estimates of energy intake with total energy expenditure determined by the doubly labeled water method in young children. Journal of American Dietetic Association92,11404.

10. Kennedy, G., Ballard, T. \& Dop, M. (2013). Guide pour mésurer la diversité alimentaire au niveau du ménage et de l'individu. Rapport de l'Organisation des Nations Unies pour l'alimentation et l'agriculture (FAO). 56 p.

11. Lawn, J. \& Harvey, D. (2003) La nutrition et la sécurité alimentaire à Kugaaruk, au Nunavut. Livre $96 \mathrm{p}$.

12. Atwater W.O., Benedict F.G. (1902). Experiments on the metabolism of matter and energy in the human body, 1898-1900. United States. Office of experiment stations. Bulletin no. 109. Government printing office, Washington, DC.https://doi.org/015962/bhl.title.123031.

13. Déré K.A.L., Djohan Y..F, Koffi K.G.., Manhan K., Niamké AG. \& Tiahou G. (2016). Individual dietary diversity score for diabetic and hypertensive patients in Cote d'Ivoire. International Journal of Nutrition 1(4) 25-34. 
14. CNN (Conseil National de Nutrition). (2015). Analyse de la situation nutritionnelle en côte d'ivoire. Rapport 2015. 78p.

15. Sanginga, N. \& Mbabu, A. (2015). Racines et Tubercules (Manioc, Igname , Pomme de Terre et Papate Douce). Centre International de Conférence Abdou Diouf Dakar, Sénégal. 35 p.

16. Macauley, H. \& Ramadjita, T. (2015). Les cultures céréalières: riz, maïs, millet, sorgho et blé. Centre International de Conférence Abdou Diouf Dakar, Sénéga 138 p

17. Shiraishi, Y., Takagi, Y., Hagi, S., Sato, A., Saeki, R., Bakaypko, K. $\&$ Tanoh, T. (2016). Enquête sur les produits cuisinés et dérivés de Tsumiré / Surimi ( produits emballés sous vide).Rapport de mission en Côte d'Ivoire. 102-20.

18. Ruf, F. (2010) “'Les Baoulé ne sont pas des oiseaux pour manger $d u$ riz”. Alimentation migrations et écologie du sud-ouest ivoirien', $1 \mathrm{p}$.

19. Dubresson, Al. (1989) 'Urbanisation et consommation alimentaire citadine en Côte d'Ivoire', 190(1), pp. 3-8.

20. N'Zué B., Zohouri G. P., Djédji C. \& Tahouo O. (2013). Bien cultiver le manioc en Côte d'Ivoire. Rapport du Centre National de Recherche Agronomique. 4p.

21. Guy-grand, B. (2017). Dernière minute. Cahiers de nutrition et de diététique. 9960(17), pp. 30029-9.

22. N'dri-Yoman, 2014. Politique nationale de prévention et de prise en charge des maladies chroniques non transmissibles en Côte d'Ivoire. Rapport du Ministère de la Santé et de la Lutte contre le SIDA, 30 p.

23. Sangaré A., Koffi E., Akamou F. \& Fall C.A. (2009). État des ressources phytogénétiques pour l'alimentation et l'agriculture: Second rapport national. Rapport national sur l'état des ressources phytogénétiques pour l'alimentation et l'agriculture, $65 \mathrm{p}$.

24. Bricas N, \& Attaie H. (2009). La consommation alimentaire des ignames. Synthèse des connaissances et enjeux par la recherche. L'igname, plante séculaire et culture d'avenir, Cirad, Inra, Orstom, Coraf, Coll Colloques, pp.21-30.

25. Kouamé, G. \& Enoh, G. (2011). Dynamique de la consommation alimentaire en Côte d'Ivoire : principales tendances. $4 \mathrm{p}$. 
ANNEXES : PHOTOGRAPHIES POUR ENQUETE

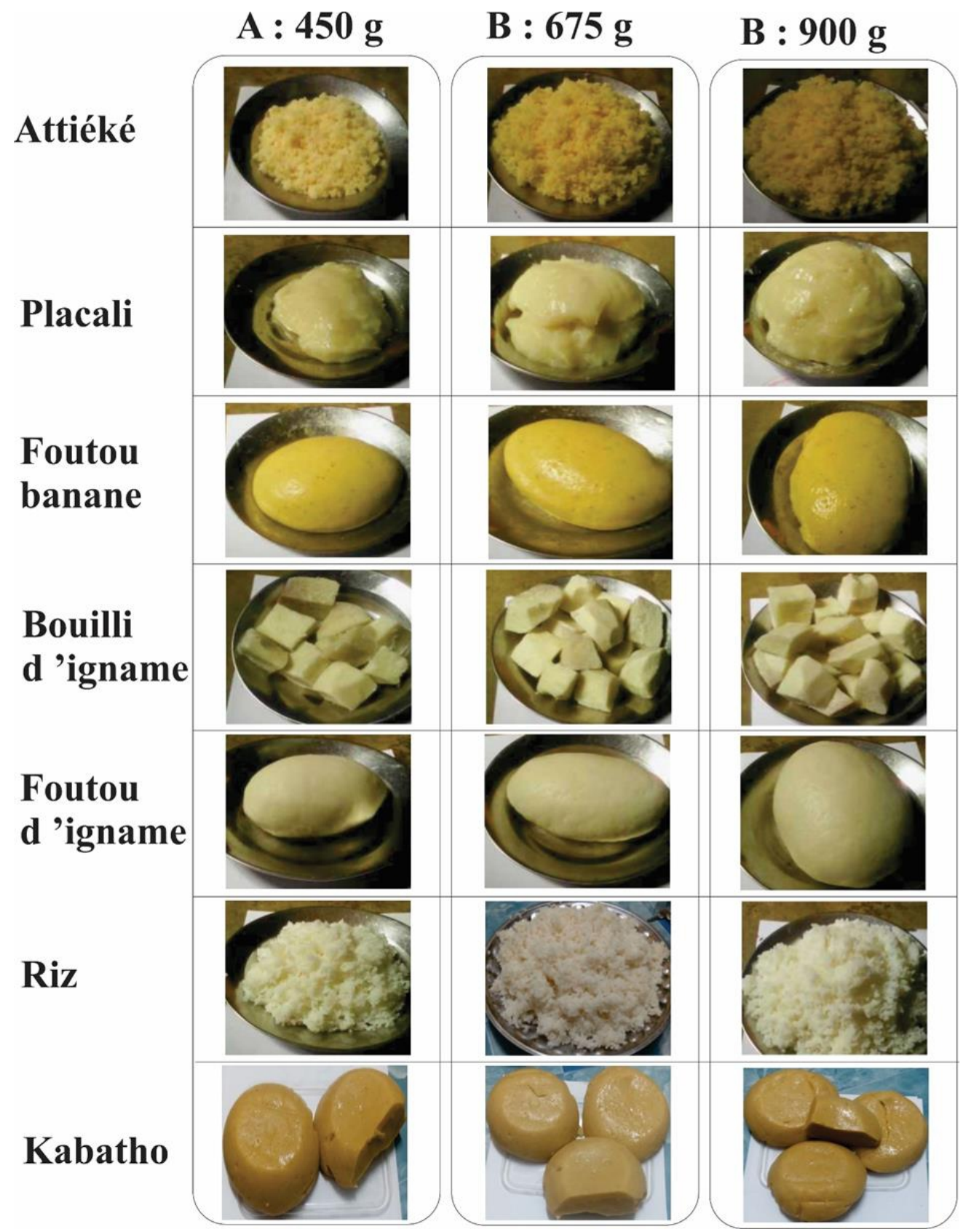

